\title{
Le déficit des précipitations dans le Sud de la France (pluie-neige) pendant l'hiver 1988-1989. Conséquences sur l'hydrologie de haute et moyenne montagne
}

\author{
D. Duband, P. Tourasse, J. Pinte \\ EDF-DTG - Grenoble
}

Au mois de mars 1989, on constatait dans le Sud de la France, un déficit important des précipitations depuis la fin d'automne et pendant l'hiver essentiellement dans les Alpes du Sud y compris la vallée de l'Arc et la Vanoise ainsi que dans les Pyrénées et le Massif Central malgré des averses neigeuses de 50 à $150 \mathrm{~mm}$, selon les régions, en fin février. En conséquence, l'enneigement en haute et moyenne montagne présentait un déficit assez exceptionnel puisque l'on observait $30 \%$ du stock normal au $1^{\mathrm{er}}$ mars, le Beaufortin et les Alpes du Nord étant moins défavorisés avec $50 \%$ du stock normal. Cette situation n'a pas empiré avec les précipitations normales de mars, et s'est même améliorée progressivement du fait des précipitations très excédentaires d'avril, qui ont atténué le déficit en eau de certaines régions, mais ont pu engendrer un optimisme prématuré quant aux incidences sur les écoulements du printemps suivant.

L'analogie avec les situations pluviométriques observées en 1949 et en 1921 à la même époque de l'année est frappante bien que les situations météorologiques à l'origine de ces pénuries soient différentes. Depuis 40 ans c'est l'un des hivers les plus secs. Et lorsqu'on se réfère à la dizaine de séries historiques ayant cent ans et plus d'observations, la durée de retour moyenne de cet événement est comprise entre 30 et 100 ans, en première estimation.

En corollaire, l'hydraulicité des hautes vallées pyrénéennes et alpines de Haute-Provence et côtières était particulièrement faible.

\section{Aperçu météorologique}

Avant d'étudier la pluviométrie et la nivométrie proprement dites, revenons sur le fait météorologique marquant du début de l'hiver 1988-1989. La persistance de très hautes pressions atmosphériques pendant 2 mois (du 20 décembre au 20 février) sur les Alpes et, plus généralement, sur le Sud de l'Europe occidentale apparaît en effet comme un événement exceptionnel. Ceci peut être résumé dans la comparaison (fig. 1) du champ de pression moyen du mois de janvier 89 à la normale des 35 dernières années et au mois de janvier 1964, référence de cet échantillon en la matière.

En 1964, l'anticyclone avait intéressé une plus vaste zone, s'étendant aux Iles Britanniques; en 1989, les Iles Britanniques et la Scandinavie ont été soumises à un flux d'Ouest intense, tandis que les pressions étaient exceptionnelles sur les Alpes (moyenne mensuelle la plus élevée à Lyon d'après une série de 110 ans).

Enfin on ne peut passer sous silence l'événement qui a marqué la fin de cette longue période. Fin février, une gigantesque dépression a envahi l'Europe Occidentale. Le 25 , la pression à Paris est tombée à $948 \mathrm{hPa}$, battant le record historique du 24/12/1821 $(959 \mathrm{hPa})$. Nous comparons (fig. 2) cette situation à celle du 3 décembre 1976, jour où les plus faibles pressions des 35 dernières années avaient été observées.

Après un mois de mars normal, le mois d'avril s'est caractérisé par des précipitations exceptionnellement fortes

The precipitation deficit in the south of France (rain and snow) during the winter of 1988-89. Consequences on the hydrology of high and medium-high mountains

A detailed spatial analysis of winter falls and the snow cover in mountain areas (Jura, Massif Central, Pyrénees) in relation with the contributions in the upper basins of the Rhone, Loire, Dordogne and the Garonne and their affluents for the period 1948-88, puts the hydro-climatological events of the hydrological year 1988-89 into context.

A duration and spatial extension comparison of these water shortages, in relation to the dry years of the last hundred years $(1880,1921,1942,1949,1976 \ldots)$ has been carried out by studying the longest historical series available in France. Is it an event that is part of the variability of our present climate, or can it be seen as the first sign of climatic change? 

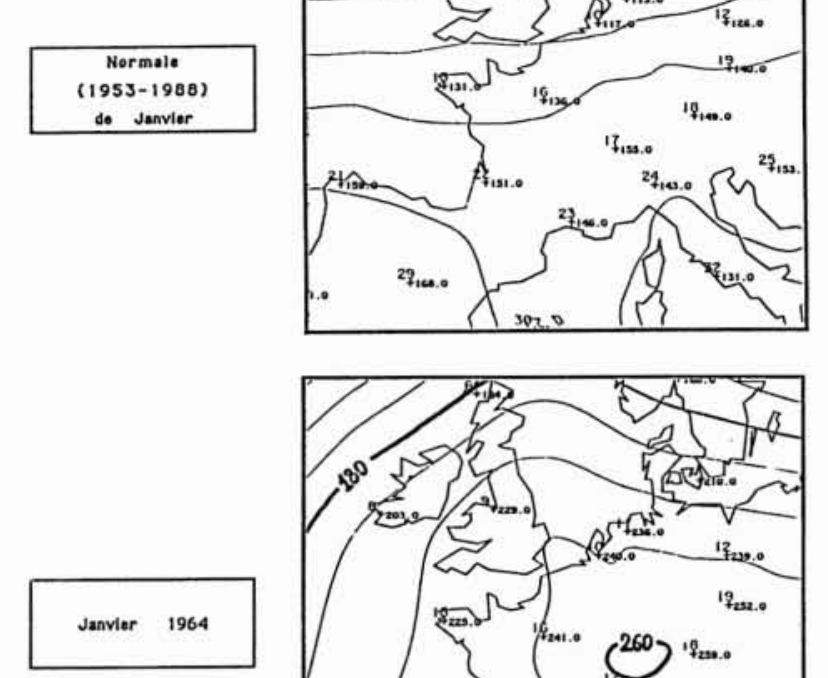

1.
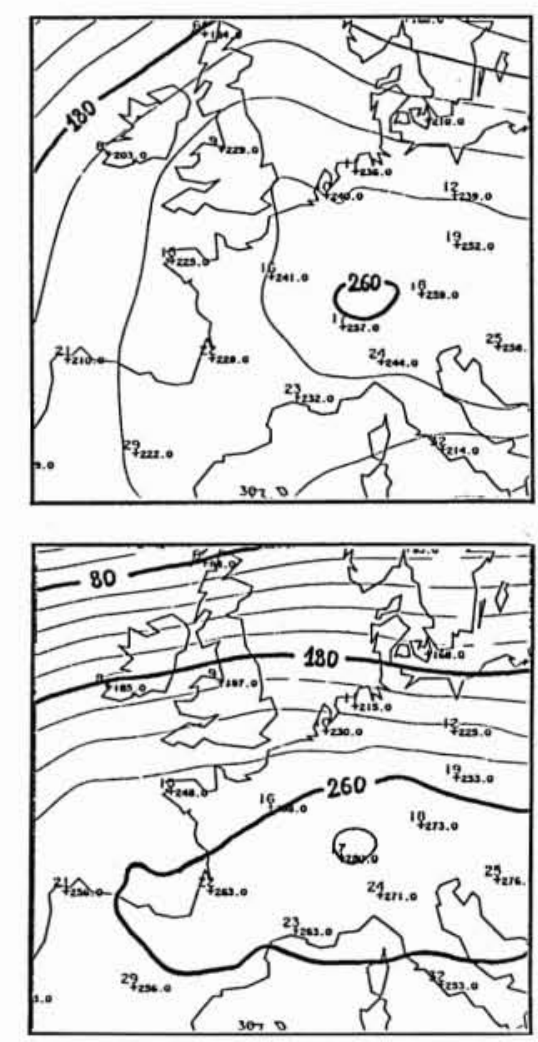

Comparaison de la situation du 26 Février 1989 avec celle du 3 Décembre 1976

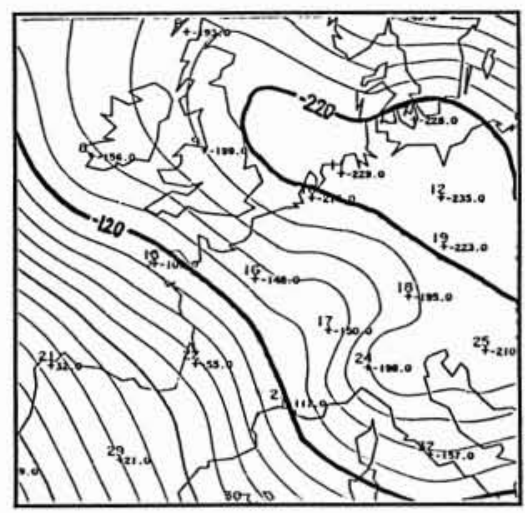

26 Ftvrier 1989

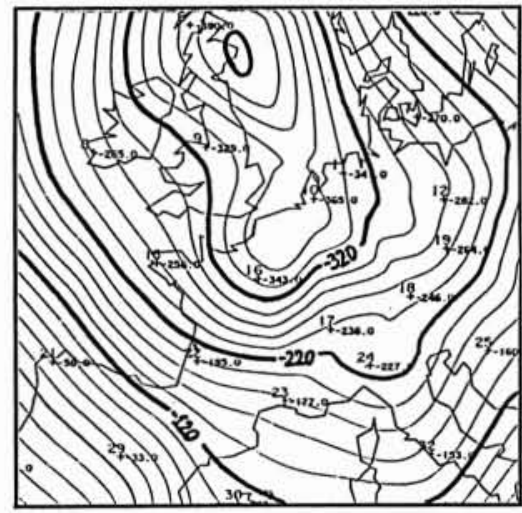

1. et 2. Isophyses de la couche $1000 \mathrm{hPa}$. pouvant atteindre 100 à $300 \%$ de la normale selon les régions. Cependant le mois de mai voit le retour du déficit pluviométrique.

\section{Précipitations et enneigement d'hiver et de prin-} temps dans les Alpes et le Jura

\subsection{Evolution}

Au cours de l'hiver et du printemps $88-89$ les précipitations enregistrées sur le Jura et l'ensemble des Alpes ont été marquées par le caractère nettement déficitaire de la période $1^{\text {er }}$ novembre-20 février notamment sur le HautArc, la Haute-Durance, le Verdon et l'ensemble des fleuves côtiers, puis, sur l'ensemble des bassins cette fois, par le caractère au contraire très excédentaire des précipitations du mois d'avril.

En mai, les précipitations ont été à nouveau très déficitaires en toutes zones avec des températures nettement supérieures à la normale qui ont provoqué une fusion rapide du manteau neigeux.

\subsection{Analyse du déficit pluviométrique}

Les distributions de précipitations cumulées de novembre à février, de novembre à mars et novembre à avril ont été établies pour la plupart des stations du Jura et des Alpes dont les données sont disponibles sur la période commune 1948-1986. Trois d'entre-elles sont données à titre d'exemple : Marigny $(465 \mathrm{~m})$ sur l'Ain, Granscala $(1720 \mathrm{~m})$ près de la frontière italienne du Mont-Cenis et Coursegoules $(1000 \mathrm{~m})$ dans l'arrière-pays niçois. Elles permettent de 

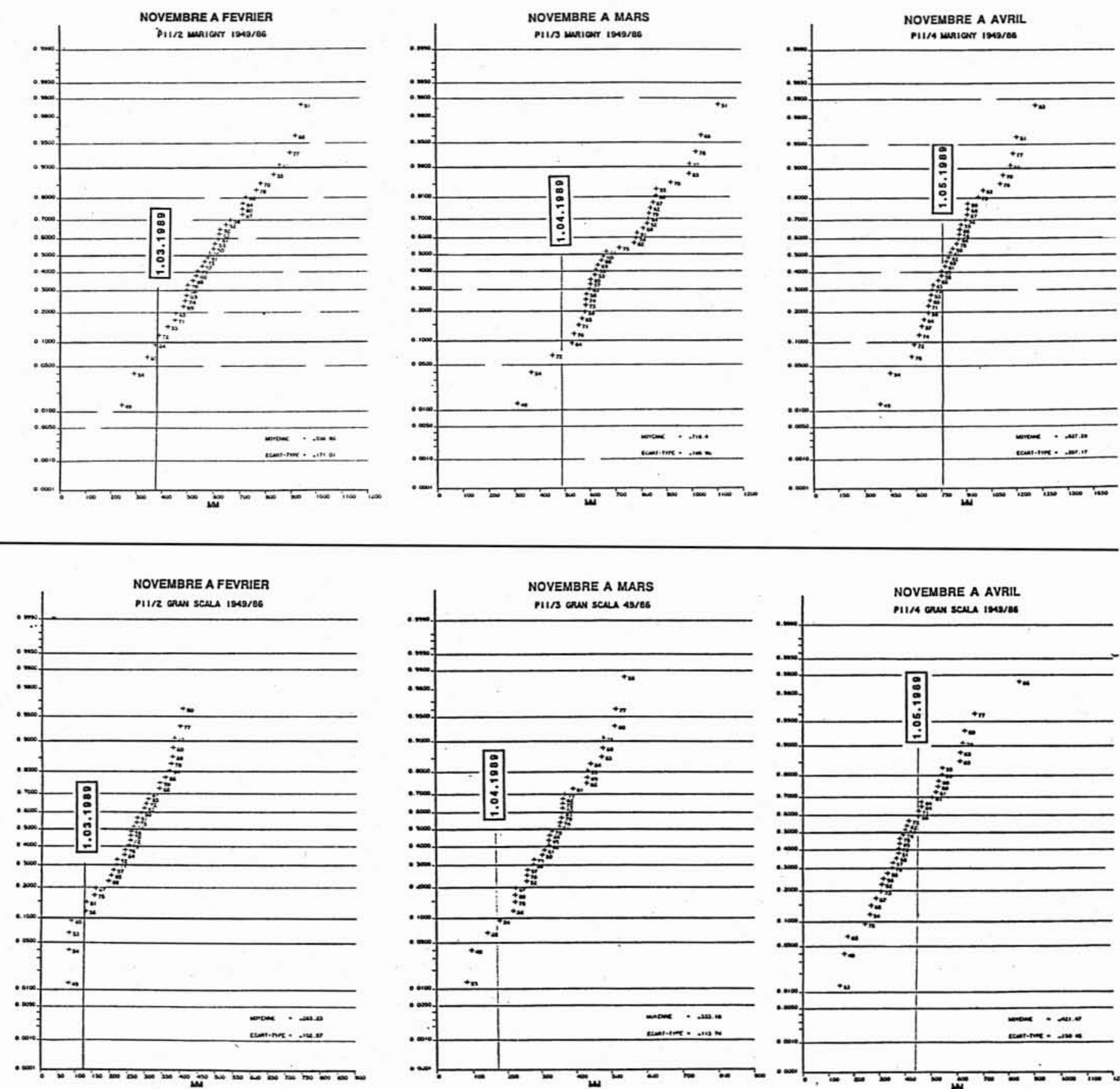

4
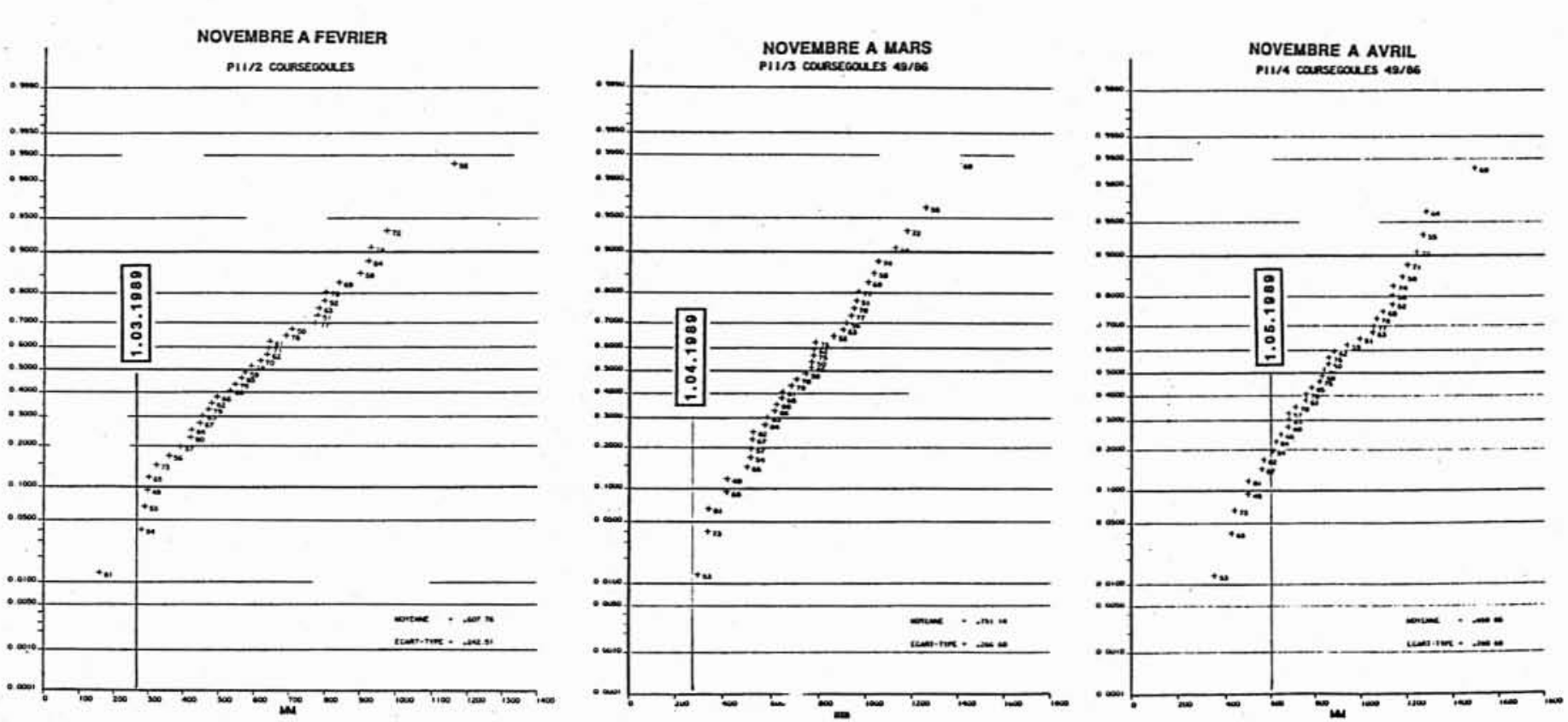
positionner les cumuls de l'hiver 89 avant et après les précipitations très excédentaires du mois d'avril (fig. 3, 4, 5).

Sur le Nord, l'évolution, en terme de fréquence, est plus sensible pour le Jura, la Haute-Isère ou la haute vallée de l'Arc puisque l'on passe d'un déficit quasi décennal au $1^{\mathrm{er}}$ avril à une situation qui, en terme de précipitations cumulées, devient normale (Marigny) voire légèrement excédentaire (Granscala) au $1^{\text {er }}$ mai. Cette évolution est encore plus remarquable si l'on compare la situation du $1^{\text {er }}$ mai 89 à celle du $1^{\text {er }}$ mars lorsque la durée de retour de la sécheresse de l'hiver 88-89 pouvait alors encore être estimée à 50 ans à Taninges (DuBAND et GARCON, 8 mars 1989 - Grenoble - Section de glaciologie - SHF).

Dans les Alpes du Sud, le caractère exceptionnel des précipitations d'avril ( 250 à $300 \%$ de la normale) n'est pas suffisant pour revenir à une situation normale au $1^{\mathrm{er}}$ mai.

Ainsi, sur la Haute-Durance, après un déficit par rapport à la normale de $70 \%$ au $1^{\text {er }}$ mars (total des précipitations cumulées de novembre à février) et de $60 \%$ au $1^{\text {er }}$ avril - dates auxquelles le déficit est comparable à celui de 1949 - le déficit au $1^{\text {er }}$ mai reste notable et atteint encore $30 \%$ de la normale.

De même, plus au Sud dans les Alpes-Maritimes, le total des précipitations des 5 mois de novembre à mars présente un déficit maximum de $70 \%$ au $1^{\text {er }}$ avril et de fait, après un mois d'avril particulièrement excédentaire (maximum des 40 dernières années enregistré à Coursegoules, $300 \%$ de la normale sur l'ensemble de la zone) le déficit pluviométrique au $1^{\mathrm{er}}$ mai reste plus que quinquennal dans tout ce secteur, les précipitations déficitaires du mois de mai n'ayant fait qu'accentuer cette situation.

3.4.5. Distribution des précipitations d'hiver cumulées.
3. Marigny (Ain).
4. Gran Scala (Haut-Arc).
5. Coursegoules (Arrière-pays niçois).

6. Chroniques de l'enneigement observé entre le I janvier et le 30 juin.

\subsection{Enneigement}

Avant le 20 février l'enneigement de moyenne et haute altitude était très déficitaire sur l'ensemble des massifs des Alpes et du Jura. Il ne représentait, à cette date, que 20 à $35 \%$ du stock normal de fin d'hiver dans les massifs les plus défavorisés (Vanoise, Haut-Arc) des Alpes du Nord et moins de $10 \%$ du stock normal de fin d'hiver dans les Alpes du Sud, soit la plus faible valeur connue depuis plus de 30 ans.

Après les précipitations de fin février qui ont fait progresser le stock de 15 à 25 points sur l'ensemble des
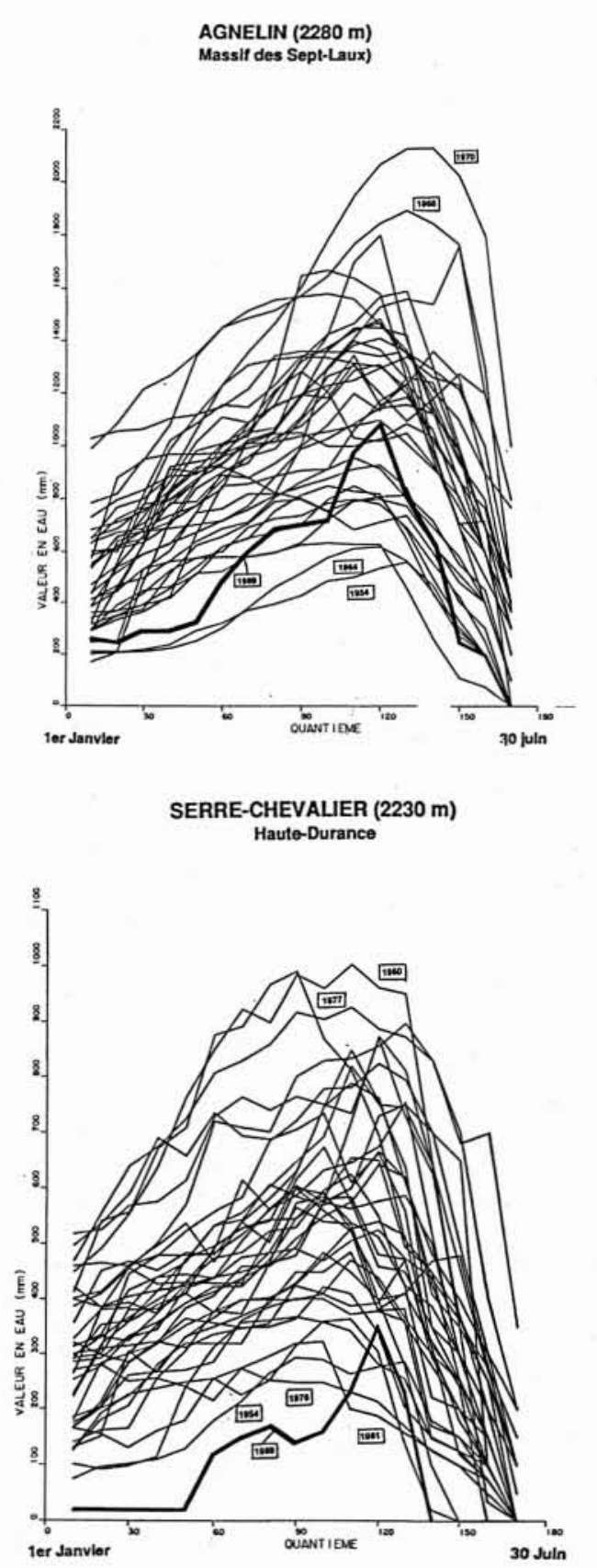

6.

Alpes puis celles du mois de mars qui ont surtout touché les Alpes du Nord, la situation nivométrique reste très déficitaire au $1^{\text {er }}$ avril sur l'ensemble des Alpes.

Dans la moitié Nord le stock à cette date ne représente encore que 60 à $70 \%$ de la normale sur les massifs avancés de l'axe Belledonne-Beaufortin et à peine 40 à $50 \%$ dans la haute vallée de l'Arc.

Il en est de même dans la haute vallée de la Durance (fig. 6) où l'on observe encore à cette date le plus faible enneigement depuis 1952 (45\% de la normale) ainsi que sur le Verdon et la zone frontalière où le déficit reste encore plus important ( 15 à $20 \%$ seulement de la normale). 


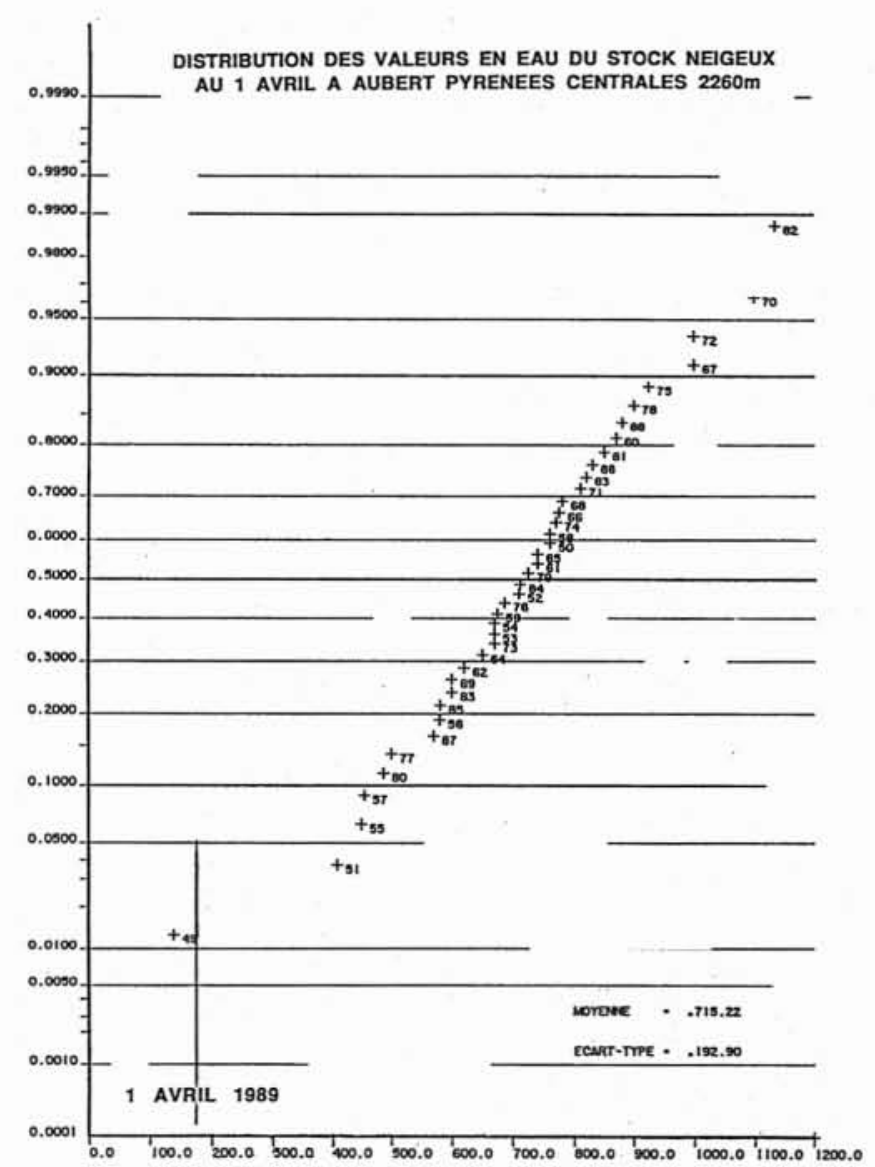

7.

Après les précipitations excédentaires du mois d'avril, l'enneigement dans les Alpes a atteint des valeurs plus proches de la normale dans les massifs intra-alpins des Alpes du Nord et s'améliore nettement dans la haute vallée de l'Arc où le gain, près de la frontière italienne, atteint pratiquement 50 points.

Dans les Alpes du Sud, malgré une progression sensible, le stock d'altitude reste encore très déficitaire. Il ne représente au $1^{\text {er }}$ mai que $70 \%$ de la normale de fin d'hiver sur la Haute-Durance et encore à peine $40 \%$ sur le HautVerdon.

\section{Précipitations et enneigement dans les Pyrénées et Massif Central}

L'hiver 88-89 a été marqué sur l'ensemble du Sud-Ouest et du Massif Central par un déficit important en précipitations jusque fin mars. L'hiver 89 peut être comparé à l'hiver 49 à la suite duquel avait sévi une sécheresse très prononcée. Même si les précipitations d'avril ont été en toutes régions, largement supérieures à la normale, excepté dans les Pyrénées-Orientales, les conditions d'une sécheresse d'été sont propices.

\subsection{Evolution}

Après une fin d'été et un automne peu humides, l'hydraulicité est inférieure à la normale sur tous les bassins. Le déficit pluviométrique s'accentue dès la mi-novembre pour atteindre son paroxisme fin février sur le Massif Central et fin mars sur les Pyrénées.

Ce déficit pluviométrique s'est traduit sur le Massif Central par des écoulements d'hiver largement déficitaires à l'époque des hautes eaux annuelles. A Bort sur la Dordogne il ne s'écoule que $141 \mathrm{~mm}$ de novembre à février alors que la moyenne est de $350 \mathrm{~mm}$ soit un déficit de $60 \%$.

Sur les Pyrénées, le stock neigeux est exceptionnellement faible au $1^{\text {er }}$ avril, ne représentant que 20 à $30 \%$ de la normale suivant les bassins: à la perche d'Aubert (2 $270 \mathrm{~m}$ ) dans le Massif du Néouvielle le stock neigeux est de $170 \mathrm{~mm}$ en 1989 (130 mm en 1949) alors que la moyenne (49-88) de la série est de $715 \mathrm{~mm}$ (fig. 7). L'espérance d'écoulement de fusion est donc très faible et largement dépendante des précipitations de printemps.

\subsection{Analyse régionale}

Pyrénées: Les observations de la période novembre 88 avril 89 pour l'Aude à Puyvalador montrent que les rares précipitations de décembre vont constituer un faible stock neigeux qui évoluera peu jusqu'à la mi-mars (températures froides et stationnaires en altitude). La hausse importante des températures de la fin mars provoque une fusion totale du stock jusqu'à 1800 voire $2000 \mathrm{~m}$ sur les versants Sud. Au $1^{\text {er }}$ avril le stock à la Gaougetta $(2050 \mathrm{~m})$ est de $250 \mathrm{~mm}$ alors que la moyenne est de l'ordre de $1200 \mathrm{~mm}$ à la même date, soit un déficit de $80 \%$. A la Quillane $(1700 \mathrm{~m})$ le stock est quasiment nul durant tout l'hiver ; au $1^{\text {er }}$ avril le déficit est de $100 \%$ (fig. 8,9 ).

Au-dessus de $2250 \mathrm{~m}$ le faible stock s'est relativement bien conservé jusqu'au $1^{\mathrm{er}}$ avril. Il est évalué au $1^{\mathrm{er}}$ avril à 20 à $30 \%$ de la normale suivant les bassins. Les fonds de vallée et le bassin de l'Aude, d'ordinaire sensible aux pénétrations des perturbations remontant d'Espagne sont les plus touchés par le déficit. A Troumouze $(2050 \mathrm{~m})$, au fond de la vallée du Gave de Pau, le stock au $1^{\text {er }}$ avril est de $245 \mathrm{~mm}$ alors que la moyenne est de $914 \mathrm{~mm}$ soit un déficit de $75 \%$. Les précipitations largement excédentaires d'avril ( $165 \%$ de la normale en moyenne) plus marquées sur les fonds de vallée et les températures plutôt froides ont contribué à recréer un stock tardif évalué au $1^{\mathrm{er}}$ mai à $60 \%$ de la normale de fin d'hiver.

La première quinzaine de mai est chaude et sèche et la limite d'enneigement est remontée à $2000 \mathrm{~m}$ au 15 mai.

Massif Central: Les observations pour la Truyère à Grandval $\left(1782 \mathrm{~km}^{2}\right)$ montrent que le stock neigeux est quasiment nul jusque fin février où de fortes précipitations neigeuses à moyenne altitude $(250 \mathrm{~mm}$ en 5 jours sur le Cantal) vont constituer un stock important mais éphémère sur l'Ouest du massif (Dordogne, Lot-Truyère, Tarn). Ce stock disparaît dans la première quinzaine de mars engendrant une forte hydraulicité et une reconstitution partielle des réserves hydrauliques. 

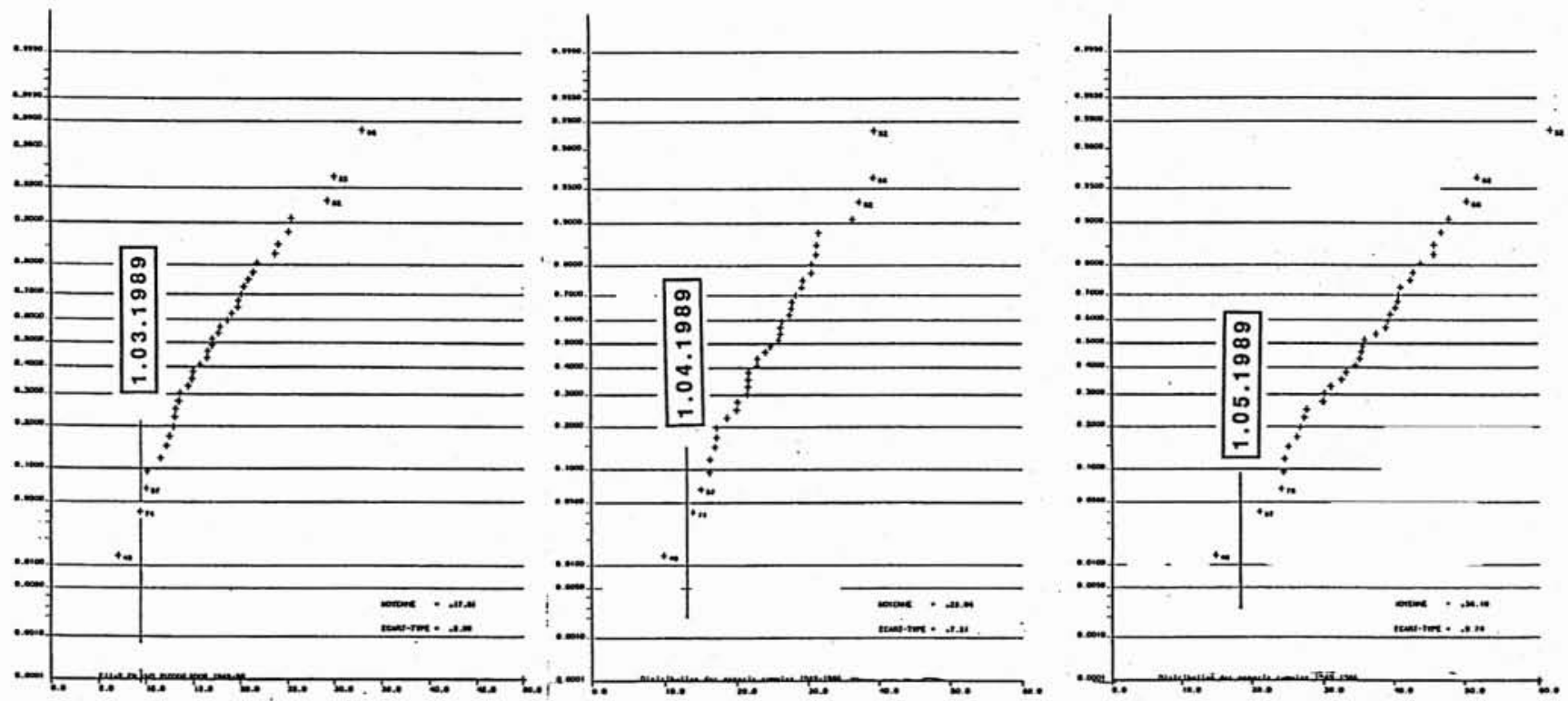

8. Distribution des apports d'hiver cumulés. Puyvalador $-134 \mathrm{~km}^{2}$ (Aude).

NOVEMBRE A FEVRIER P11/2 AUDE 49/86

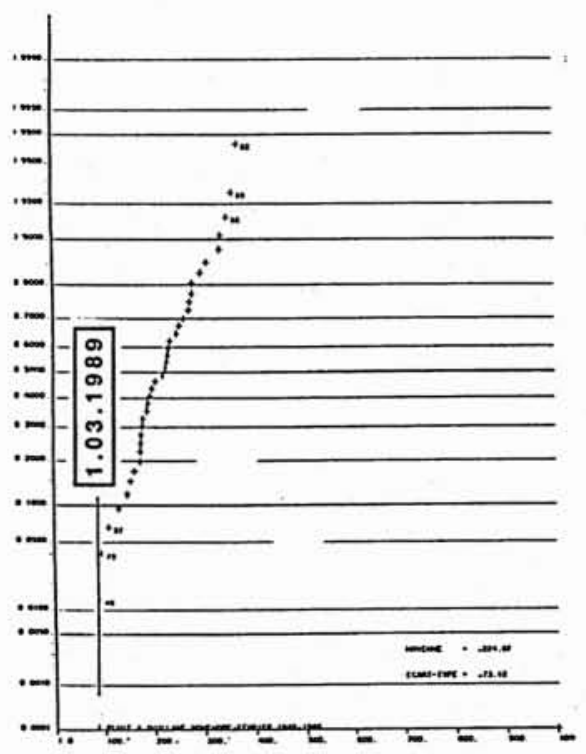

NOVEMBRE A MARS

P11/3 AUDE 49/86

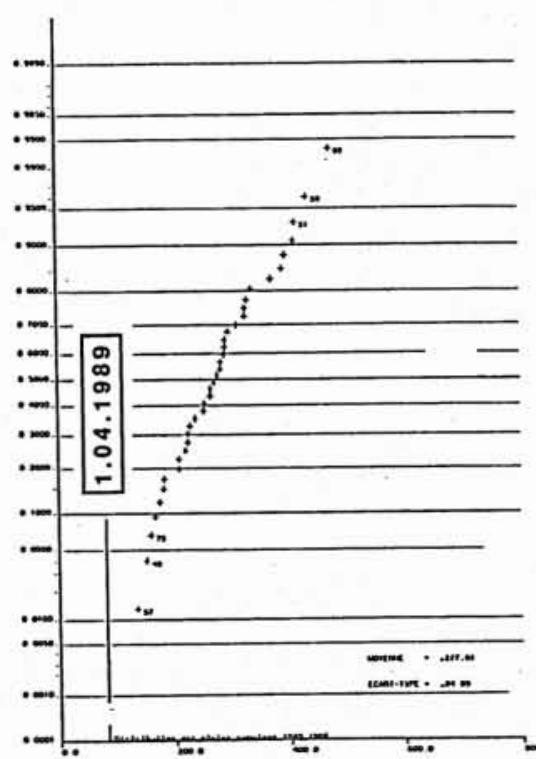

NOVEMBRE A AVRIL P11/4 AUDE 49/86

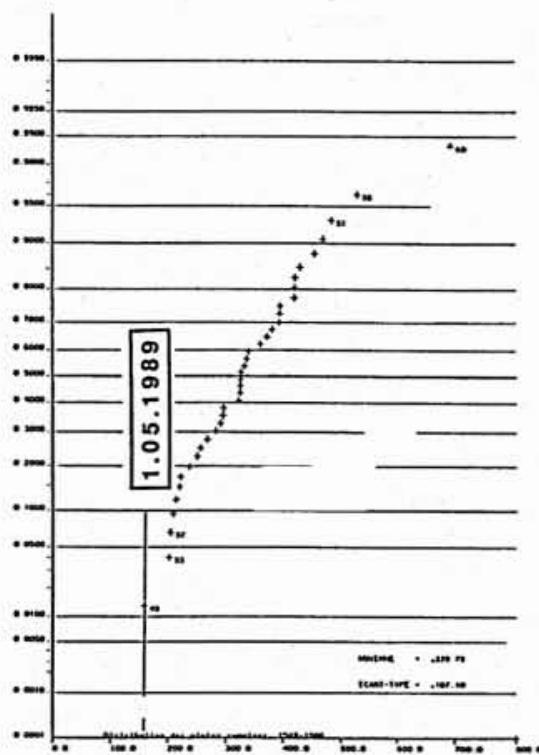

9. Distribution des apports d'hiver cumulés. La Quillane (Aude).

En revanche, fin mars, le débit des cours d'eau atteint à nouveau et très rapidement le quantile $10 \%$ ce qui traduit le fait que les réserves souterraines ne sont pas reconstituées et que le déficit de l'hiver est très marqué.

Avec des précipitations d'avril très excédentaires (170 à $230 \%$ de la normale du Nord au Sud) le déficit d'écoulement s'atténue d'autant que la crue des 25-26 avril, géné- ralisée sur le Sud-Ouest du massif, a largement participé à l'hydraulicité supérieure à la normale du mois.

En revanche sur le Nord Massif Central (Creuse à Eguzon $2400 \mathrm{~km}^{2}$ et Dordogne à Bort $1010 \mathrm{~km}^{2}$ ) le déficit persiste à la fin avril : sur la distribution des écoulements cumulés de novembre à avril, l'année 1989 reste comparable à l'année 1949 . 


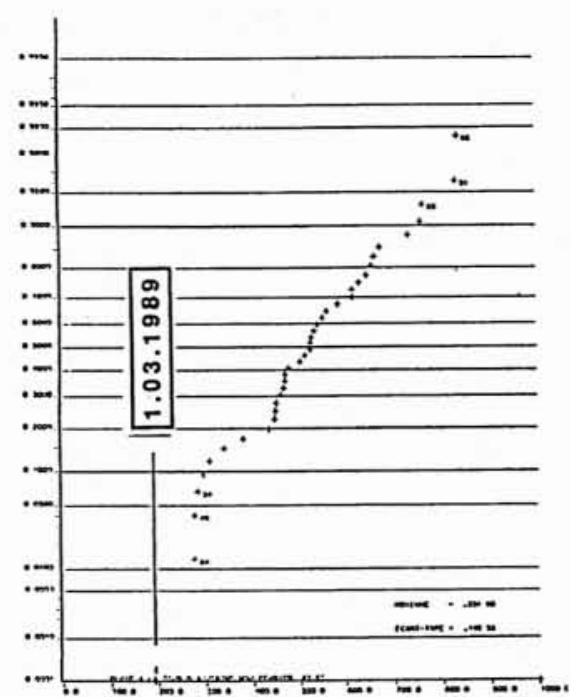

NOVEMBRE A MARS P11/3 TOUA D'AUVERGNE 49/86
NOVEMBRE A AVRIL P11/4 LA TOUR D'AUVERGNE 49/86
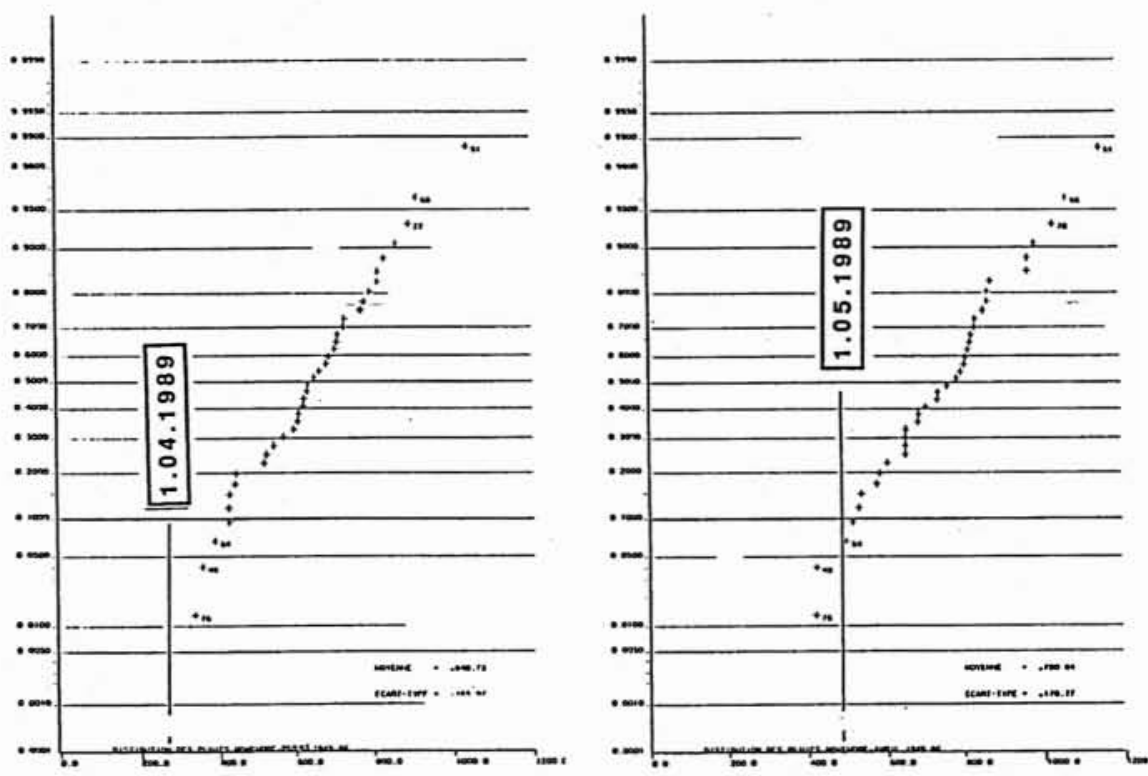

10. Distribution des précipitations d'hiver cumulées. La Tour d'Auvergne (Dordogne)

NOVEMBRE A FEVRIER

E11/2 CAMON 49/86
NOVEMBRE A MARS

E11/3 CAMON 49/86
NOVEMBRE A AVRIL E11/4 CAMON 49/86
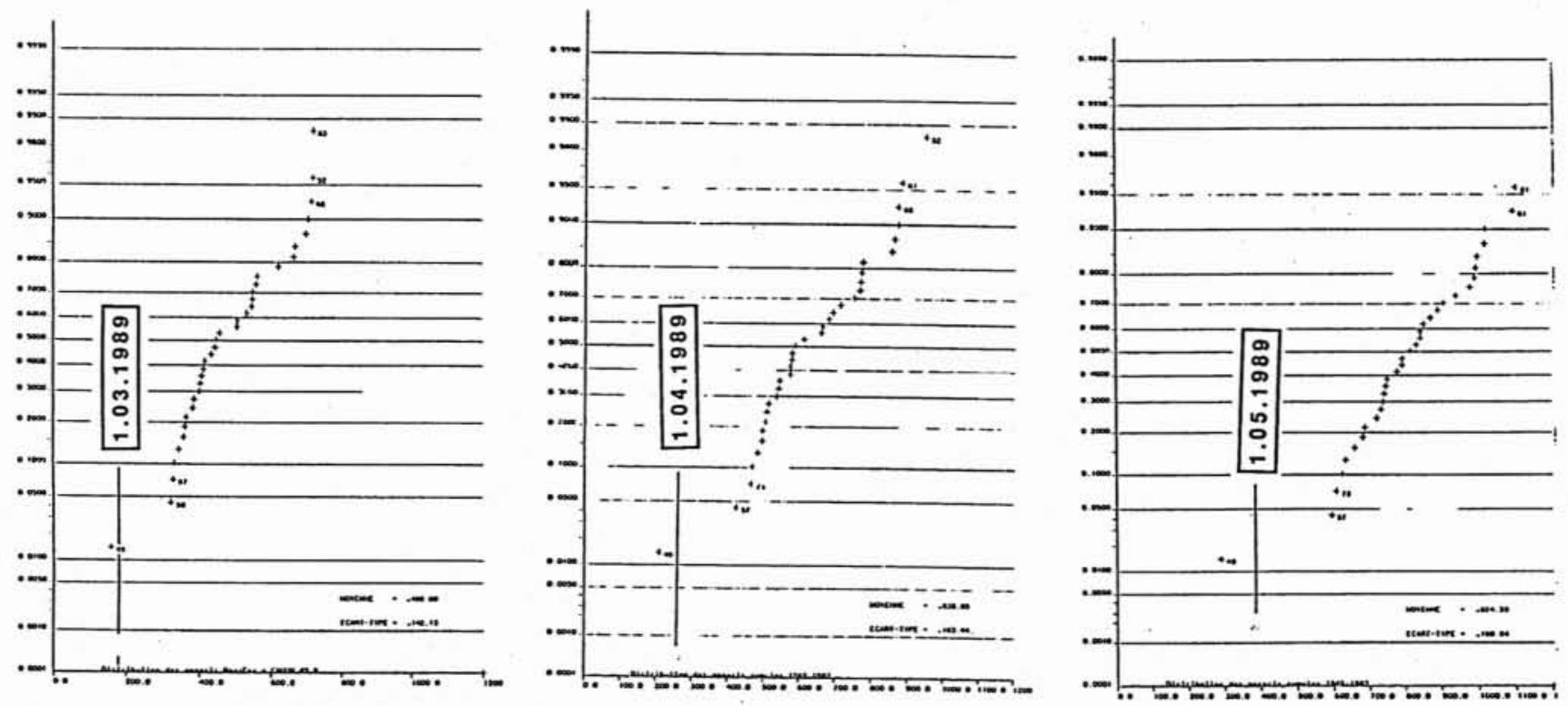

11. Distribution des précipitations d'hiver cumulées. Camon - $2230 \mathrm{~km}^{2}$ (Garonne).

\subsection{Analyse de la situation au $1^{e r}$ mai}

Au regard des distributions des écoulements cumulés $\left(\mathrm{E}_{11-\mathrm{i}}\right)$ de novembre à février, à mars, à avril et des distributions de précipitations $\mathrm{P}_{11-\mathrm{i}}$ cumulées de novembre à février, à mars, à avril, il est clair que le déficit est maximum au $1^{\text {er }}$ avril en toute région (fig. 10).

\begin{tabular}{|c|c|c|c|c|c|c|c|c|}
\hline STATION & $\begin{array}{l}\text { COURS } \\
\text { DEAU }\end{array}$ & $\begin{array}{c}\text { BASSIN } \\
\text { VERSANT }\end{array}$ & $\begin{array}{l}\text { Ell-2 } \\
\text { en mm }\end{array}$ & $\% \mathrm{~N}$ & $\begin{array}{l}\text { El1-3 } \\
\text { en mm }\end{array}$ & $\% \mathrm{~N}$ & $\begin{array}{l}\text { Ell-4 } \\
\text { enmm }\end{array}$ & $\begin{array}{l}\% / N \\
49-86\end{array}$ \\
\hline Pont d'Escot & Gave d'Aspe & $450 \mathrm{~km} 2$ & 129 & 21 & 241 & 30 & 420 & 43 \\
\hline Camon & Garonne & $2230 \mathrm{~km} 2$ & 81 & 55 & 112 & 53 & 171 & 57 \\
\hline Puyvalador & Aude & $134 \mathrm{~km} 2$ & 68 & 51 & 96 & 51 & 133 & 49 \\
\hline St-Peyres & $\mathrm{Am}$ & $129 \mathrm{~km} 2$ & 122 & 20 & 253 & 32 & 442 & 50 \\
\hline $\begin{array}{l}\text { Pinet } \\
\text { Pins }\end{array}$ & Tam & $2677 \mathrm{~km} 2$ & 61 & 20 & 126 & 33 & 205 & 45 \\
\hline Sarrans & Truyère & $2462 \mathrm{~km} 2$ & 38 & 15 & 133 & 41 & 235 & 60 \\
\hline Bort les Orgues & Dordogne & $1010 \mathrm{~km} 2$ & 141 & 22 & 241 & 30 & 363 & 40 \\
\hline Equzon & Creuse & $2400 \mathrm{~km} 2$ & 69 & 35 & 78 & 31 & 123 & 42 \\
\hline
\end{tabular}

L'année 1949 demeure l'année de comparaison de référence.

C'est fin mars que le déficit pluviométrique est le plus accusé en toute région (fig. 11). Sur les Pyrénées cette constatation est à rapprocher des très faibles stocks au $1^{\text {er }}$ avril (Aubert à $2250 \mathrm{~m}$ au cœur du massif du Néouvielle : $170 \mathrm{~mm} \mathrm{au} 1^{\mathrm{er}}$ avril avec une durée de retour supérieure à 100 ans).

\begin{tabular}{|l|l|r|r|r|r|r|r|r|}
\hline \multicolumn{1}{|c|}{ STATION } & BASSIN & \multicolumn{1}{|c|}{ ALT } & $\begin{array}{r}\text { P11-2 } \\
\text { en mm }\end{array}$ & $\%$ N & $\begin{array}{r}\text { P11-3 } \\
\text { en mm }\end{array}$ & \%N & $\begin{array}{r}\text { P11-4 } \\
\text { en mm }\end{array}$ & $\begin{array}{r}\text { \%/N } \\
49-86\end{array}$ \\
\hline Arnouste & Gave doOssau & $1140 \mathrm{~m}$ & 170 & 27 & 219 & 28 & 415 & 45 \\
Eget & Garonne & $1016 \mathrm{~m}$ & 88 & 23 & 141 & 31 & 312 & 58 \\
La Quillane & Aude & $1714 \mathrm{~m}$ & 81 & 36 & 93 & 33 & 159 & 47 \\
Florac & Tam & $550 \mathrm{~m}$ & 83 & 19 & 117 & 22 & 307 & 50 \\
St-Chély d'Apcher & Truycre & $1000 \mathrm{~m}$ & 73 & 27 & 107 & 33 & 266 & 68 \\
\hline La Tour d'Auvergne & Dordogne & $970 \mathrm{~m}$ & 189 & 36 & 260 & 40 & 484 & 64 \\
\hline
\end{tabular}




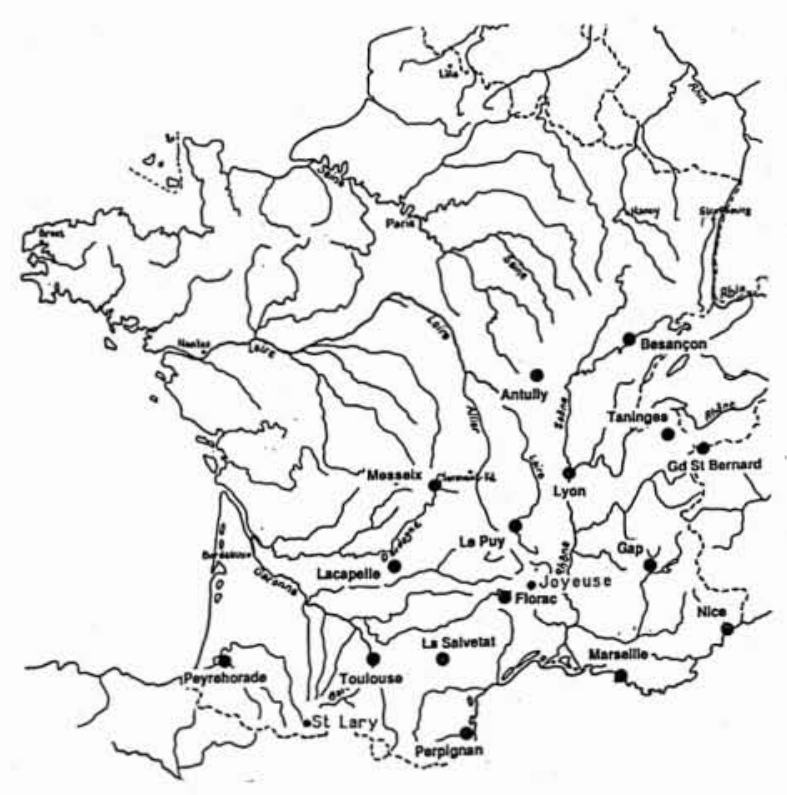

12. Analyse statistique des longues séries pluviométriques. Localisation des 18 stations retenues.
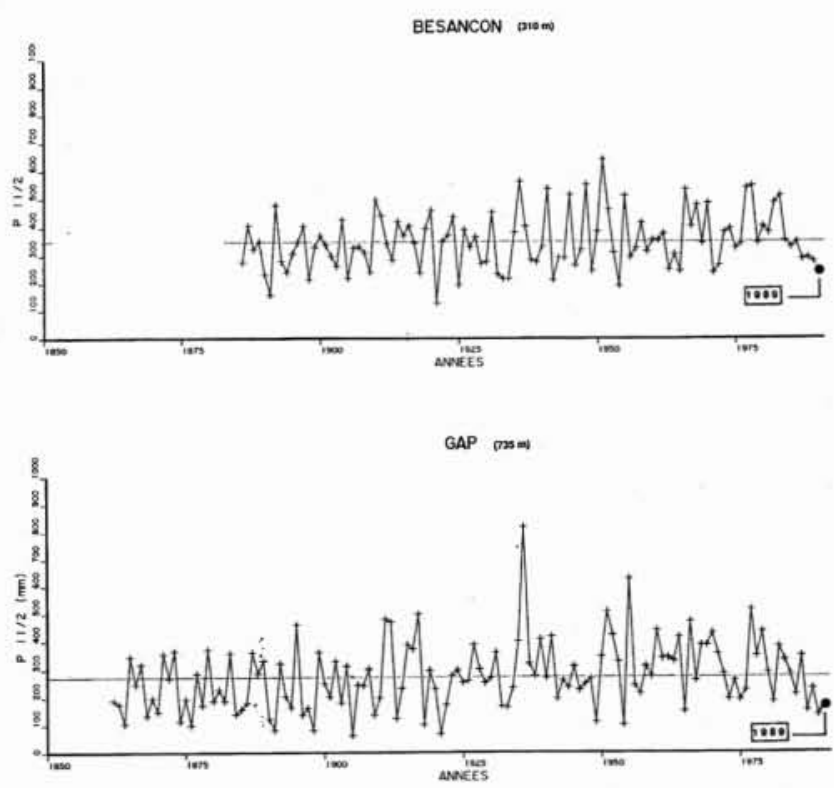

13. Série des précipitations cumulées de novembre à février.

\subsection{Nuances et perspectives}

Dans les Pyrénées, compte tenu des précipitations neigeuses d'avril, les écoulements de ce mois ont été relativement faibles ( 60 à $80 \%$ de la normale) ce qui maintient le déficit d'écoulement au $1^{\text {er }}$ mai.

Sur les Pyrénées Centrales et Occidentales (Gave d'Aspe à Pont d'Escot et Garonne à Camon) l'année 1989, en terme d'écoulement est plus critique que l'année 1949 au $I^{\text {er }}$ avril $\left(\mathrm{E}_{11-3}\right)$ et demeure du même ordre de grandeur au $1^{\text {er }}$ mai $\left(E_{11-4}\right)$. La durée de retour du phénomène est au moins cinquentennale. Les prévisions d'apport de fusion de mai à juillet laissent espérer au mieux 60 à $80 \%$ des apports normaux suivant les bassins si les précipitations neigeuses d'avril ont un rendement normal et si les précipitations de printemps sont elles-mêmes normales.

Sur le Massif Central le déficit pluviométrique atteint son maximum fin février et persiste jusqu'au $1^{\text {er }}$ avril malgré l'épisode important d'Ouest qui arrose tout le massif fin février.

L'épisode pluvieux généralisé (excepté au Nord du Massif) des 25-26 avril a amené un ruissellement important mais il faut noter qu'après chaque épisode pluvieux la décrue et le tarissement qui suivent sont rapides. Au 15 mai, les débits du Massif Central sont moitié de ce qu'ils étaient au $1^{\text {er }}$ mai. Il semble donc que l'alimentation profonde des nappes soit réduite, conséquence du déficit d'hiver. Avec un mois de mai peu pluvieux les conditions de sécheresse d'été sont latentes.

\section{Analyse de la rareté du déficit depuis le début du siècle}

L'acuité de la sécheresse nous a obligé, pour estimer sa fréquence, à retenir les séries pluviométriques les plus longues. Nous avons sélectionné 18 stations réparties assez régulièrement sur une zone englobant les Pyrénées, le Massif Central, les Alpes et le Jura et pour lesquelles nous disposions de plus de 70 ans de données. Ces points de mesure sont localisés sur la figure 12.

A titre indicatif, la chronologie des précipitations d'hiver à Gap et Besançon est représenté d'après plus d'un siècle d'observation à la figure 13.

Analyse locale: Les distributions des cumuls de précipitations de novembre à février ont été tracées. Deux d'entre elles sont données figure 14 (page suivante) avec la position de l'hiver 89 avant et après les pluies de fin février.

Les durées de retour de la sécheresse de l'hiver 88-89 peuvent être estimées à 10 ans à Gap, 30 ans à Marseille, 40 ans à Peyrehorade, 50 ans à Taninges, plus de 50 ans à Messeix et Florac, alors qu'elles n'atteignent pas 10 ans à Perpignan, Besançon et Antully.

Les millésimes les plus souvent rencontrés à l'extrémité de ces distributions sont les années 1921 et 1949.

Analyse globale: Pour synthétiser les résultats locaux, on a effectué une analyse en composantes principales sur les cumuls novembre-février des 18 stations de 1913 à 1984 . 

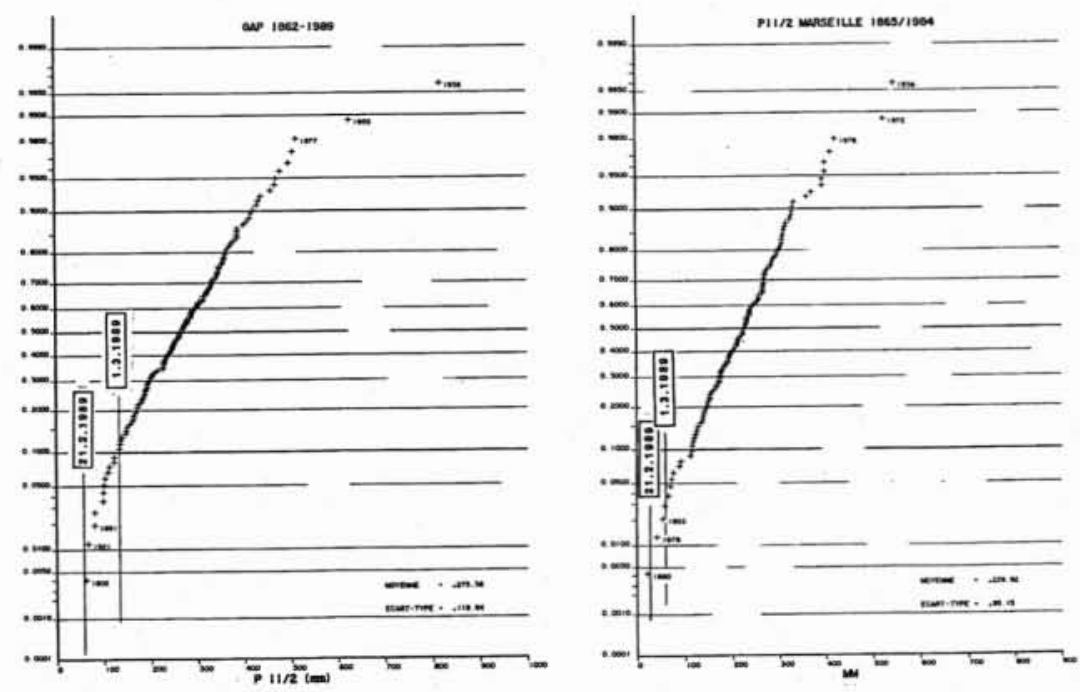

14. Distribution des précipitations cumulées de novembre à février.
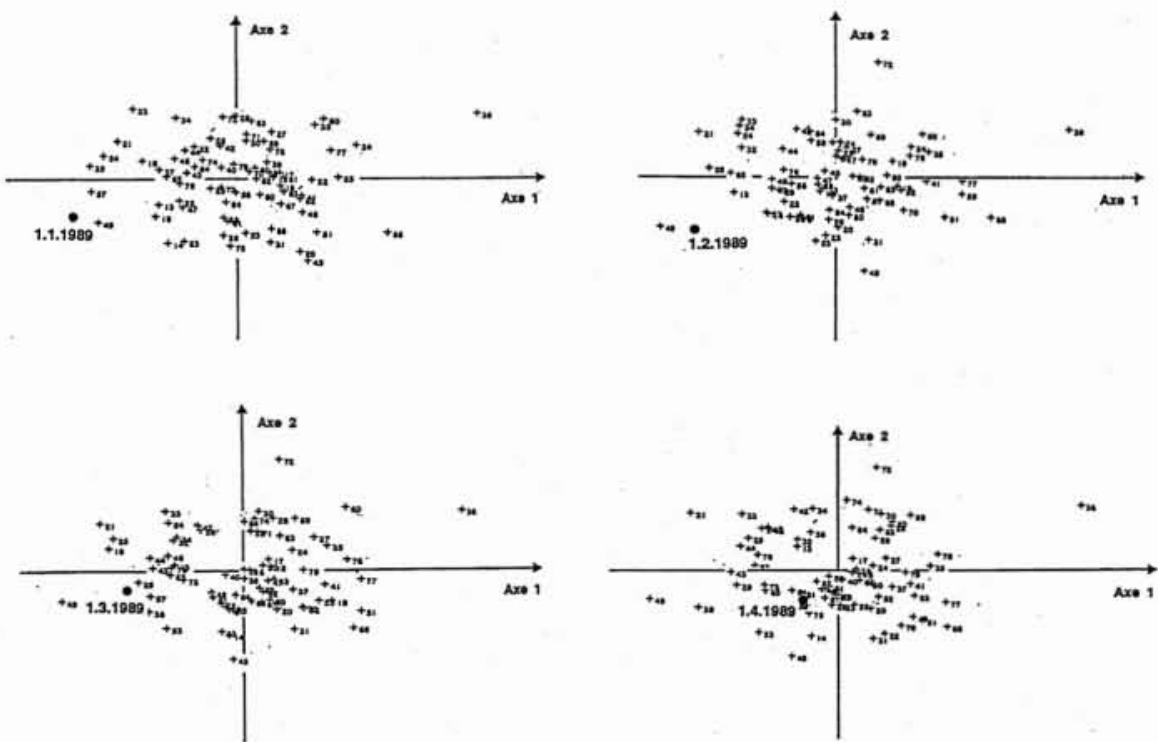

15. Position des hivers 1913 à 1984 sur le premier plan factoriel.

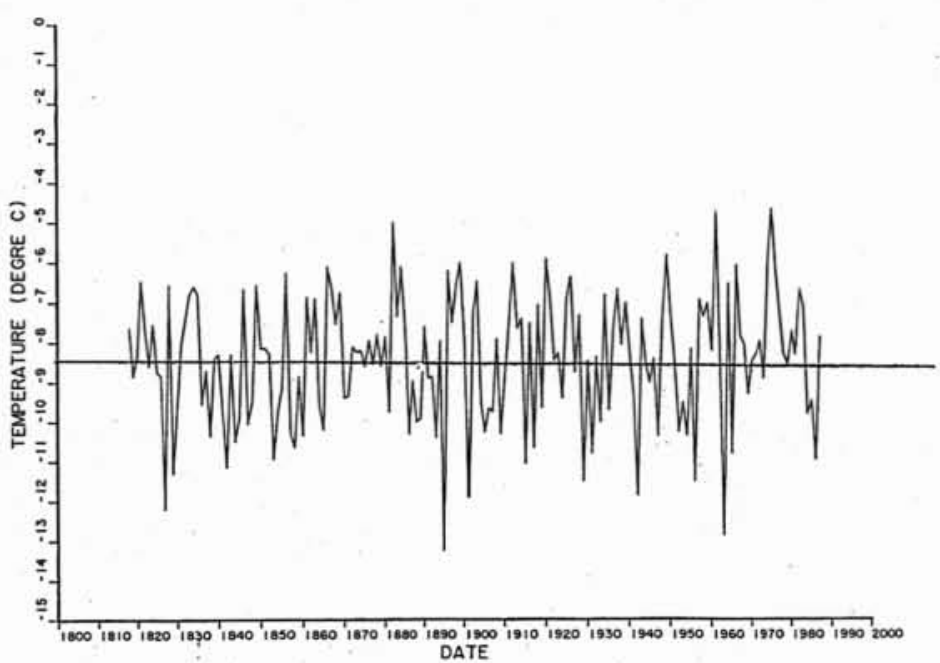

16. Grand St-Bernard $(2479 \mathrm{~m})$. Températures de l'air. Moyennes janvier-février de 1818 à 1987. 


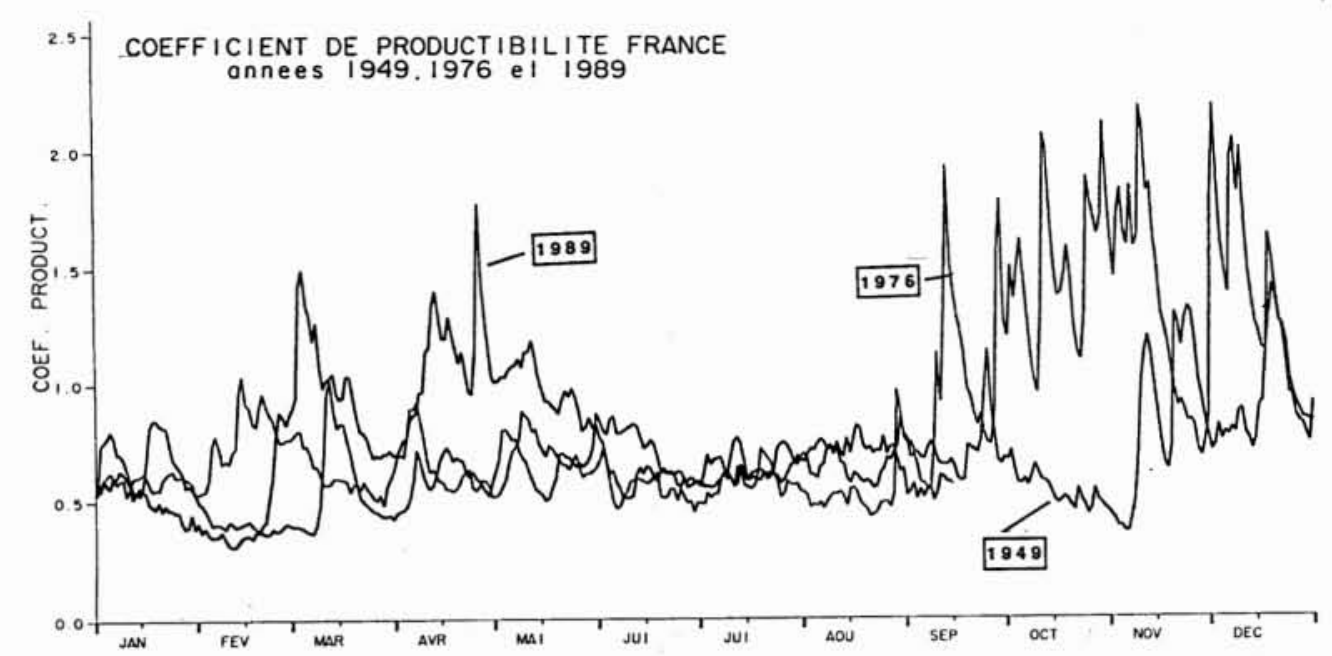

17.

Le premier facteur est assimilable à un facteur de taille : Perpignan, témoin d'un climat particulier, est la seule station qui soit cotée négativement sur cet axe. Le second axe oppose les régions exposées aux précipitations de Nord-Ouest à celles plus sensibles aux flux méridionaux.

Sur le premier plan factoriel qui contient $72 \%$ de l'information totale, les stations sont disposées progressivement selon leurs directions principales d'alimentation : de l'exposition Ouest, Nord-Ouest (Morvan, Jura, Alpes du Nord), on passe par l'Ouest (façade Ouest du Massif Central), le Sud-Ouest (Peyrehorade, Toulouse) puis le Sud (Florac, Marseille), pour atteindre finalement l'Est (Perpignan).

La position des observations sur le $\mathrm{I}^{\mathrm{er}}$ Plan factoriel met en évidence les hivers réputés secs, notamment 1920-1921 plus spécialement déficitaire sur l'alimentation de NordOuest et 1948-1949 avec des déficits plus importants sur les alimentations de Sud et de Sud-Ouest.
On a également fait figurer la situation de l'hiver 19881989 au $1^{\text {er }}$ janvier, $1^{\text {er }}$ février, $1^{\text {er }}$ mars, $1^{\text {er }}$ avril (fig. 15). Si l'effet de la dernière semaine de février est tangible, la situation au $1^{\text {er }}$ mars 1989 reste très voisine de celle de 1949.

L'étude chronologique et fréquentielle des précipitations cumulées d'hiver d'après les quelques séries d'observation dépassant un siècle, qui met en évidence la rareté d'occurrence de l'événement $88-89$, ne suggère pas de façon significative que notre environnement, à l'échelle de l'ouest de l'Europe, soit engagé dans un processus de modification climatique. On observe bien des alternances de séquences d'années sèches et humides sans qu'il n'y ait une quelconque régularité cyclique, avec nos connaissances actuelles. Ces constatations sont aussi valables pour les plus longues séries de températures moyennes mensuelles de l'air, Suisses, Françaises et Allemandes que nous avons pu étudier (fig. 16).

\section{Conclusion}

La situation nivo-pluviométrique en mai est préoccupante dans les Pyrénées, Alpes du Sud et le Nord du Massif Central par son analogie à celles de 1949 et 1921. Mais la comparaison s'arrête là pour le moment car les précipitations des prochains mois (juin, juillet, ...) non prévisibles dans l'état actuel des connaissances météorologiques peuvent être excédentaires, auquel cas, on peut espérer une hydraulicité s'approchant de la normale ; mais si elles sont déficitaires, nous serons en situation d'étiage au moins centennal.

En évitant tout catastrophisme pour les prochains mois, on peut cependant remarquer que le rendement des précipitations de printemps diminue avec la croissance de la végétation, et que les stocks neigeux constitués tardivement en haute montagne ont un rendement plus réduit dans leur contribution aux eaux de ruissellement. Il faut donc s'attendre à des répercussions de la sécheresse sévère des mois de novembre à mars sur le comportement des nappes phréatiques et en conséquence sur certains cours d'eau du Sud de la France pendant les mois d'été et peut être de l'automne 1989.

Enfin les séries historiques de précipitations cumulées ainsi que les températures d'hiver ne font pas apparaître de tendance particulière qui indiquerait un début de modification climatique, significative dans l'état actuel des analyses. L'hiver 88-89 apparaît comme une péripétie hydrométéorologique plausible inscrite dans les aléas du climat actuel.

Nota : Au moment de l'impression de cet article, la sécheresse s'est confirmée pendant les mois d'été dans le sud de la France, le graphique ci-joint (fig. 17) représente l'évolution comparée du coefficient de productibilité hydraulique des aménagements de l'EDF en 1989, 1976 et 1949. C'est un excellent témoin de l'hydraulicité des rivières de haute et moyenne montagne y compris le Rhône dans la moitié sud du pays. 